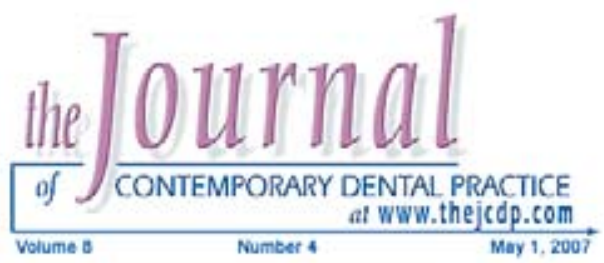

\title{
Transcultural Barriers and Cultural Competence in Dental Hygiene Practice
}

\author{
Salim Rayman, RDH, BS, MPA; \\ Khalid AImas, BDS, MSc, FRACDS, FDSRCS, DDPH, FICD, FAAOM, FADI
}

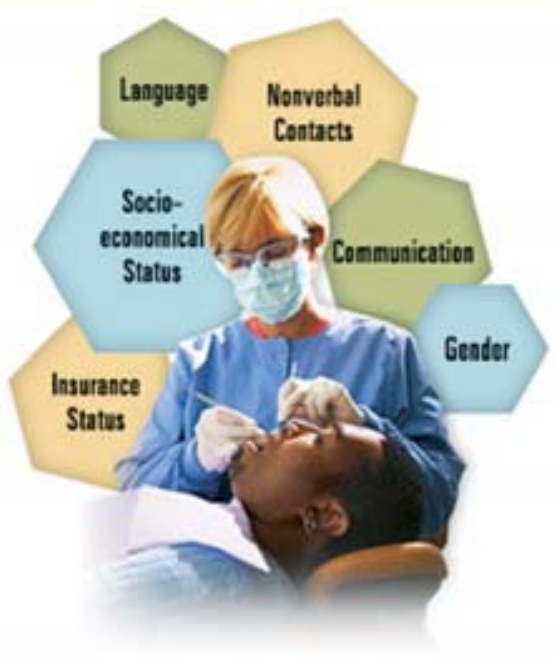

Abstract

Aim: The aim of this paper is to highlight the need to integrate cultural care into dental hygiene practice.

Background: Culture can be better understood through the concepts, beliefs, and norms of the culturally diverse population of the United States. It is important to understand cultural perceptions of oral health in order to manage transcultural barriers that may arise during the utilization of oral healthcare services. It is assumed cultural competence can only be achieved through the knowledge of various cultural backgrounds in an extremely diverse US population.

Conclusions: There is a need to integrate cultural awareness, knowledge, identification, and respect into the dental hygiene curriculum through the incorporation of competencies and standards associated with dental hygiene practice. Future dental hygienists should be culturally competent to render oral care to an ever expanding and diversified US population.

Keywords: Transcultural barriers, diversity, cultural competence, dental hygiene curriculum

Citation: Rayman S, Almas K. Transcultural Barriers and Cultural Competence in Dental Hygiene Practice. J Contemp Dent Pract 2007 May;(8)4:043-051.

(C) Seer Publishing 


\section{Concepts and Definitions of Health and Disease}

In order to achieve a deeper understanding of the cultural, social, and ethnic beliefs regarding oral health and disease, oral health and disease need to be defined. Only then can a comprehensive portrayal of oral health beliefs and cultural phenomena affecting oral health be visualized.

The World Health Organization (WHO) defines health as a "state of complete physical, mental, and social well being and not merely the absence of disease." The American Heritage Dictionary $(1976)^{2}$ defines health as follows:

1. The state of an organism with respect to functioning, disease, and abnormality at any given time.

2. The state of an organism functioning normally without disease or abnormality.

3. Optimal functioning with freedom from disease or abnormality.

In 1860 Florence Nightingale described health as "being well and using one's powers to the fullest extent." ${ }^{33}$ Murray and Zentner defined health as "a purposeful, adaptive response, physically, mentally, emotionally, and socially, to internal and external stimuli in order to maintain stability and comfort". ${ }^{4}$ Rogers described health as "symbolic of wellness, a value term defined by culture or individual". ${ }^{5}$ Today, even experienced healthcare providers may be unable to provide a comprehensive, acceptable answer to the question, "What is health?" without the use of some form of medical jargon. Rosenstock stated health professions are becoming increasingly aware of the lack of clarity in the definition of health. ${ }^{6}$ Spector notes the framework of both education and research in the health professions continues to rely on the more abstract definitions of the word health. He further states when taken in a broader context, health can be regarded not only as the absence of disease but also a reward for "good behavior" while illness is a punishment for "bad behavior." An example of a "bad behavior" can be not brushing or flossing the teeth. ${ }^{1}$ Spector further notes these concepts of health and illness are rarely, if ever, discussed in the modern education of healthcare providers. ${ }^{1}$ Students enter healthcare with their own culturally based concept of health and illness. However, through the educational and socialization process in the professions, healthcare providers shed these beliefs while adopting a more standard definition. Spector indicates in addition to shedding these cultural beliefs, healthcare providers who do not accept the prevailing, institutional connotation of the words health and disease ${ }^{1}$ are viewed as deviant.

Disease and illness tend to be regarded as the absence of health. The American Heritage Dictionary (1976) defines illness as "sickness of body or mind. Obsolete. Evil; wickedness." Kozier provides a more contemporary definition of illness as "a highly personal state in which the person feels unhealthy or ill, may or may not be related to disease". 'Illness can be a synonym for disease or it can be a person's perception of having poor health.

Disease is an actual physical, pathophysiological process which can cause an abnormal condition of the body or mind. Illness and disease are not necessarily the same. Most people who have a disease will feel they have an illness, while others will feel perfectly healthy. A third group (although small) may claim illness although they do not actually have a disease. ${ }^{8}$ People could have caries and periodontitis and in danger of losing their tooth, yet they feel perfectly well and believe they do not have an illness. Spector defines health as the balance of the person, both within one's being - physical, mental, spiritual and in the outside world - natural, communal, and metaphysical. Illness is in the imbalance of one's being - physical, mental, and spiritual and in the outside world - natural communal, and metaphysical. ${ }^{1}$ This is the perception of health and illness in a traditional sense and the way in which it is determined within most traditional cultures. There are differing perceptions of health and illness by the healthcare provider and the patient. These differences may account for the healthcare provider's misconception that services are used inappropriately and people do not care about their health. 
Cultural Perceptions of Oral Health

Oral health involving the orofacial complex exists in biologic interrelationship with the entire body and as a result is influenced by general health. Darby and Walsh describe health as a state of well-being with both objective and subjective aspects that exist on a continuum from maximal wellness to maximal illness. ${ }^{9}$ A persons' health may change along this continuum under the influence of biologic, psychological, spiritual, social, and cultural factors that are interrelated and fluctuate over time. ${ }^{9}$ Oral health can have an effect on general health through oral infections and conversely oral health is an indicator of individual systemic health. For example, oral cancer and its complications due to antibiotic therapy is a systemic disease that can produce oral changes. According to Darby and Walsh the oral cavity is analogous to other body cavities; its health status is governed by the same physical and chemical laws and physiologic principles and can be affected by the same social, cultural, psychological, and spiritual factors as other body cavities. Oral health is defined as the oral condition resulting from the interaction of individuals with their environment, under varying levels of human needs fulfillment. ${ }^{8}$

\section{Demographic Diversity of the US Population} Healthcare providers in the twenty first century are at the cutting edge of great demographic, social, and cultural changes. Moreover, many of these changes are playing an important role in the delivery of healthcare to clients, their families, and the community. In 1990 people of color (all ethnic groups other than white) constituted 19.7\% of the population; in 2000 the percentage rose to $30 \%$ of the population and is rapidly growing. ${ }^{10}$ In 2000 the population of the United States was $281,421,906$ representing an increase of 32,712 , from 1990 with $25 \%$ of the population consisting of people of color. The White nonHispanic population in 2000 was $75.1 \%$; the Black population alone was $12 \%$; the Native American, Eskimo, and Aleut together were $0.9 \%$; and the Asian/Pacific Islander population was $3.6 \%$. The Hispanic population of any race represented is $12.5 \%{ }^{10}$

Health and Socioeconomic Factors

Age: As of April 1, 2000, 72.3 million or $26 \%$ of the U.S. population were under 18 years of age.

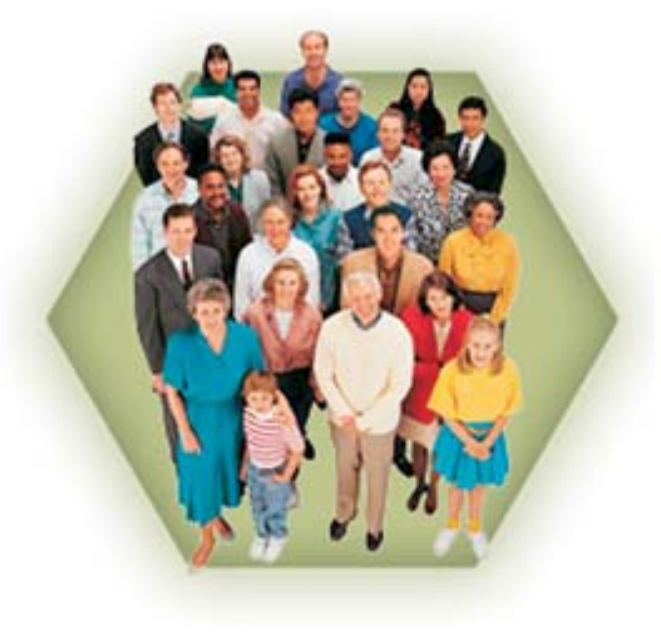

A total of 174.1 million, or $62 \%$ of the population, were between the 18 and 64 years of age. The percentage of under 18-year-old populations was greater than the total population among Blacks, American Indians, and Hispanics. A total of 35 million people or $12 \%$ were age 65 or over. The percentage of the 65-years-of-age or more population was the greatest among White nonHispanics. $^{9}$

Poverty: Poverty can be viewed from anthropological, cultural, demographic, economical, educational, environmental, historical, medical, philosophical, policy, political, racial, sexual, sociological, and theological points of view. The United States Bureau of Labor Statistics counts the poor and describes the poor by age, education, location, race, family composition, and employment status. The federal government's definition of the "poverty threshold" is based on a pretax income only, excluding capital gains, and does not include the value of noncash benefits, such as employer-provided health insurance, food stamps, or Medicaid. ${ }^{11}$ The poverty level for an average family of four was $\$ 18,100$ in $2002, \$ 8,860$ for the first person and $\$ 3,080$ for each additional person. ${ }^{12}$ The poverty rate was $11.3 \%$, meaning 31.1 million people were poor in 2000 . The Black poverty rate was $22.1 \%$; Hispanics $21.2 \%$ and the Asian poverty rate was $10.8 \%$ while White and non-Hispanics is at an all time low of $7.5 \% .^{13}$

The white majority is aging and shrinking and has the lowest poverty rate. Black, Hispanic, Asian, and American Indian populations are young, growing, and make up for the majority 
of Americans in poverty. It is imperative for healthcare providers to gain an understanding of and be sensitive to cultural differences among patients. They should also be aware of the affect these differences have on patient beliefs about health and illness and their personal practices related to their healthcare needs.

\section{Barriers to Utilization of Oral Healthcare Services}

Many problems exist within today's healthcare delivery system. Some of these are global problems affecting everyone while others are specific to the poor and to the emerging majority of populations. Spector states the healthcare delivery system fosters and maintains a childlike dependence and depersonalized condition for the consumer. ${ }^{1}$ The media and many dental companies have focused the attention of the public on cosmetic dental care to achieve the perfect smile. Advertisements advise viewers of tooth whitening products and procedures for bleaching, electric toothbrushes, and straightening teeth. At the same time, regular visits to the dental office are often overlooked even though it is critical to maintaining oral health.

One of the Healthy People 2010 objectives focuses on improving oral health and significant improvements have been made. According to the data from the National Center for Health Statistics, the percentage of adults and children with oral health problems such as dental caries and periodontal disease has decreased in recent years. ${ }^{14}$ While overall dental health has improved, dental insurance coverage has not increased. Only $44 \%$ of persons in the United States have some form of private dental insurance, most with limited coverage and with high co-payments while $9 \%$ have public dental insurance such as Medicaid and the Children's Health Insurance Program, 2\% have other dental insurance, and $45 \%$ have no dental insurance. ${ }^{14}$

Socioeconomic and insurance status has an affect on the utilization of preventive care. Poor adolescents are less likely than near-poor and non-poor adolescents to visit the dentist and to have dental sealants in place. ${ }^{15-16}$ Moreover, lowincome adolescents, especially those living near poverty, are less likely to have dental insurance $(51 \%)$ than are either their higher-income peers $(65 \%)$ or those living below the poverty level $(60 \%) .{ }^{14}$ Children and adolescents without dental insurance are three times more likely to have unmet dental needs than are children with insurance, public or private. ${ }^{13}$ However, it is important to note, according to one recent study of much younger children, access alone does not appear to account for the socioeconomic disparity in dental disease burden. ${ }^{17}$ This study surveyed first-grade children in Nova Scotia, Canada where all children are covered by one insurance program that provides basic preventive, restorative, and surgical services. Despite this universal "access"' to professional dental care, children whose parents had low educational attainment had more carious teeth than did children whose parents had completed higher levels of education. This suggests other factors such as dental hygiene behaviors and/or dietary composition also shape the socioeconomic disparities in dental health. ${ }^{16}$

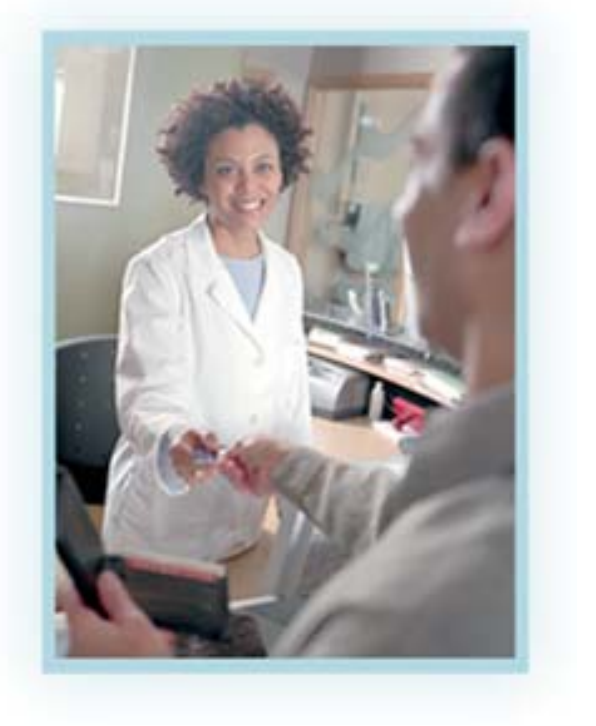

In 2002 the Community Health Data Base's Southeastern Pennsylvania Household Health Survey (SEPA) suggested lack of dental coverage and cost are principle barriers to maintaining oral health but may not be the only barriers to dental healthcare. SEPA found one out of three adults in Southeastern Pennsylvania have not been to the dentist in the past year (32.6\%), which represents approximately 937,600 adults in the region. Poor adults were more likely to have gone without dental care in the past year than non-poor adults ( $57.7 \%$ vs. $29.6 \%$ ). Minority adults are also more likely to go without dental care. Compared to $28.2 \%$ of white adults, $40.6 \%$ of Asian, $42.1 \%$ of 
Hispanic, and $44.5 \%$ of African American adults have not been to the dentist in the past year.

SEPA data indicates $33.1 \%$ do not have dental coverage; among those who do have dental coverage, many may have inadequate coverage with high co-payments and limited benefits, which may limit their access to dental care. Among the adults without dental coverage, $39.3 \%$ cited cost as the main reason why they did not visit the dentist during the past year. However, the survey found cost and lack of insurance were not the only reasons people did not visit the dentist regularly. In many cases, one of the most difficult barriers is getting adults to understand the need to prioritize dental health. According to SEPA, the adults who have dental coverage but did not go to the dentist in the past year, $25 \%$ said they "don't need [dental care]", with $13.6 \%$ stating they did not have enough time, and another $10 \%$ stating they just weren't motivated to go. Other reasons included, "not having a dentist," "just not thinking about it," or a fear of the procedure. The SEPA survey concluded adults with dental coverage are choosing not to visit the dentist regularly as well as the high percentage of adults without coverage who are not going to the dentist for reasons other than the cost. In some cases this may due to a lack of health literacy, language, cultural, or financial barriers. The survey suggested the healthcare community may not have fully educated people as to the importance of dental health and must teach adults to prioritize dental health.

\section{Transcultural Barriers to Implications of Oral Health Messages}

\section{Barriers to Utilization of Health Messages}

Effective verbal and nonverbal communications are critical aspects of all phases of the dental hygiene care process. Failure to communicate effectively and appropriately can result in misunderstanding, lack of trust, and increased anxiety levels for both the dental hygienist and client as well as the collection of inaccurate data and diagnosis, improper care planning, and treatment. ${ }^{8}$

The client and provider language disparity can be a major barrier to communication and dental care. As a result, the use of an interpreter may be necessary in cases where the client is not

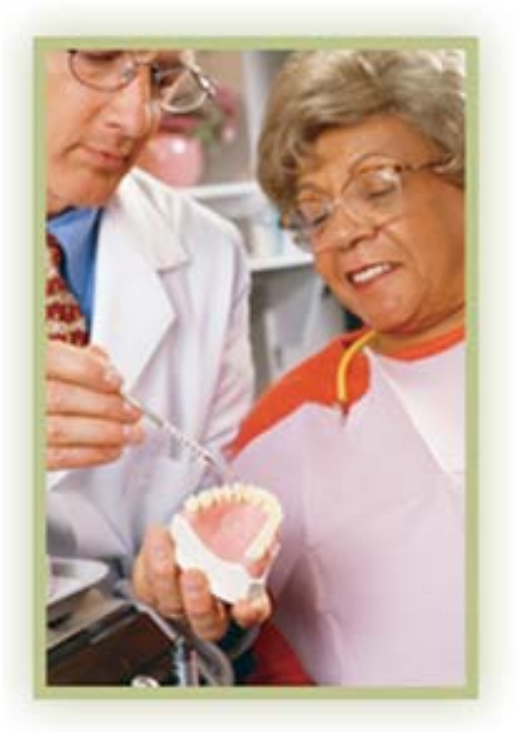

proficient in English. ${ }^{18}$ This person should be a trained interpreter and, if possible, should not be a family member. A family member may be too emotionally involved; the patient may have privacy concerns and in the process withhold sensitive information to avoid upsetting the family member. ${ }^{19}$ Moreover, the dental practitioner may find it difficult to develop a rapport with the client when using an interpreter. The practitioner should try to concentrate on looking at the client rather than the interpreter during communication and plan to pause and use extra time for translation, repetition, clarification, use of visual aids, and socialization to dental hygiene care. ${ }^{8}$ The use of open-ended questions is a means of conveying interest in a client and their needs and will produce more detailed and valuable information than using simple questions that can be answered with either yes or no. ${ }^{20}$ The dental hygienist should be aware patients from some cultures may feel it is disrespectful to ask questions, so they simply smile and nod politely. This seemingly polite behavior may be wrongfully interpreted by the practitioner as being indicative of agreement on the part of the client. ${ }^{18}$

To be successful in the mediation of cultural differences, the dental hygienist must be mindful of not only the verbal but also the nonverbal cultural variations in communication. Nonverbal communication and behaviors compromise at least $70 \%$ of all communication. ${ }^{19}$ However, use and meaning of hand gestures, acceptable physical touching, proximity, and eye contact differ depending upon cultural background and should 
be used with caution. For example, the "okay" sign used in the United States culture means "money" in the Japanese culture, "nothing" or "zero" in France, Zimbabwe, and Argentina, and it signifies a body orifice in Eastern European cultures. The "thumps up" sign is seen as a vulgar connotation in Iranian culture, and the use of a forefinger to point is viewed as rude in Chinese culture. In Middle Eastern and African cultures the left hand is seen as unclean and only used for personal hygiene and never used for handshakes and presenting gifts. ${ }^{21}$ Therefore, the dental hygienist should use less hand gestures if there is any reason to believe they could be misinterpreted by the client.

Physical touching and eye contact also varies from culture to culture. While African Americans and Hispanics are more likely to prefer close proximity when communicating, Asians, Middle Eastern, and individuals who practice the religion of Islam may not. Direct eye contact in Native Americans and Chinese cultures may be seen as a sign of disrespect, while Hispanics view this as a show of concern and attentiveness. ${ }^{20}$ To avoid these problems the dental practitioner should observe the client's verbal and nonverbal expressions for indications of discomfort and should follow the client's lead in regards to proximity and eye contact. ${ }^{1}$

Gender also plays a significant role in the utilization of dental hygiene care. In Muslim cultures, women prefer to be seen by a female healthcare provider rather than a male. Some Asian mothers prefer they and their daughters be seen by a female dentist. These behaviors are related to the value these cultures place on modesty. ${ }^{18}$ Therefore, a client should be given the option or be asked of a gender preference at the initial time of contact with the dental practitioner.

While communication, language, non-verbal contacts, and gender play a major role in the creation of barriers to dental health messages, educational status of the clients are also critical in utilization of such messages. A study of first-grade children by Ismail and Sohn ${ }^{16}$ was done in NovaScotia, Canada where all children are covered by one insurance program that provides basic preventive, restorative, and surgical services. They found despite universal access to dental care,

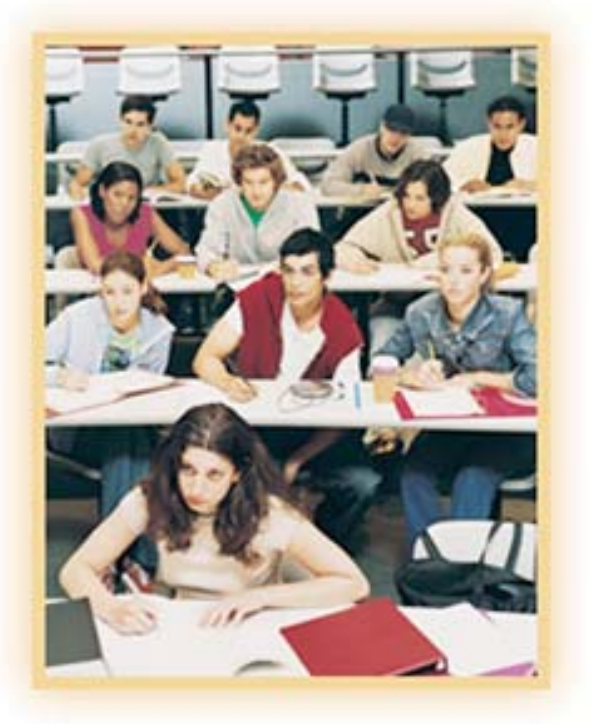

children whose parents had a low level of educational attainment had more carious teeth than did children whose parents had completed higher levels of education. The Center for Disease Control National Health and Nutrition Examination Survey of 1999 - 2002 found a correlation between the prevalence of untreated tooth decay in permanent teeth and education in adults. ${ }^{22}$ In 1999 to 2002 approximately $41 \%$ of adults with less than high school education had at least one or more untreated decayed permanent teeth compared to $14 \%$ of adults with more than a high school education. In addition, $13.5 \%$ of adults with less than a high school education were edentulous compared to $3.5 \%$ of adults with more than a high school education. ${ }^{22}$ Sundby and Petersen found almost all Danish mothers who had more than seven years of education, with children at 15 years of age, $7 \%$ of them had dental caries in their incisors or smooth surface compared to only $33 \%$ of mothers of a Turkish background with seven years or more of education, and $26 \%$ of Turkish adolescents had dental caries in incisors or smooth surfaces. ${ }^{23}$ Sundby and Petersen concluded the number of years of education received by parents, specifically mothers, was a factor for dental health in children. ${ }^{23}$ For children in particular, mothers rather than fathers of ethnic minority groups were responsible for taking care of the child's dental health. Mothers due to their lower number of years of education were not actively employed as well and did not have sufficient experience in the new language, which contributed to relatively poor communication skills and its affect on dental health. 
Cultural Competency in Education and Practice To practice effectively in a pluralistic society such as the United States, the dental hygienist cannot properly provide complete care from an ethnocentric perspective without becoming culturally and educationally competent. The dental hygiene practitioner must be able to assimilate cultural care into the dental hygiene process of care in order to provide effective, quality dental care. ${ }^{24}$ The current population demographics reveal an increasingly multicultural society; therefore, dental hygienists must be prepared to practice in a culturally sensitive and appropriate manner to deliver optimal oral hygiene care to all clients.

\section{Professional Implications in Education and Practice}

Providing oral healthcare based on the clients' individual needs is the cornerstone of dental hygiene practice. The Commission on Dental Accreditation states in order to effectively interact with and treat diverse population groups, graduating dental hygiene students must be competent in multicultural, interpersonal, and communication skills. ${ }^{25}$ The American Dental Hygienists' Association (ADHA) Code of Ethics for Dental Hygienist includes beliefs, fundamental principles, and core values requisite to the provision of cross-cultural dental hygiene care in a multicultural society. The United States Department of Health and Human Services (USDHHS) states in its Healthy People 2010 report there is a need for a "cadre of clinically and culturally competent providers" who are available to provide care where different languages, cultures, and healthcare beliefs would otherwise create barriers to care. ${ }^{26}$ According to Fitch ${ }^{23}$, in order for dental hygiene educators to teach cultural care they must first become educated themselves. The support for educating educators on cultural care is provided by the USDHHS, which recommends government support for special care training programs including cultural competence for faculty, dental students, and dental healthcare providers. Morey and Leung state dental hygiene educators need to consult with their colleagues in nursing, psychology, and cultural anthropology when planning and developing transcultural curricula. ${ }^{27}$ Dental hygienists are required to obtain continuing education credits during each licensure period, and courses in cultural competence would be a valuable option to meet this need. The ADHA endorses the concept of cultural competence and sponsoring continuing education programs addressing cultural care can also be a valuable educational option. ${ }^{23}$ In addition, dental and dental hygiene programs in local colleges and universities as well as private dental practices can provide in-service programs presented by knowledgeable, culturally competent dental hygiene educators, dentists, or consultants.

Fitch $^{23}$ states if dental hygiene cultural care is to become a reality, it is imperative dental hygiene programs actively recruit faculty and students from diverse cultural backgrounds in order to enhance the development of cultural awareness and competence. Faculty and student cultural diversity should ideally reflect population demographics. This is a difficult task because a lower proportion of minorities enroll in postsecondary institutions due to lower high school completion rates, disproportionately higher poverty rates, attendance at academically poorer elementary and secondary schools, limited access to medical care, and lack of access to educational and career opportunities. ${ }^{28}$ The dental hygiene profession has the least culturally diverse workforce of dental healthcare providers when compared to dentists, dental assistants, and dental laboratory technicians. As a result of the insufficient number of minorities entering the dental hygiene profession, there is shortage of minority dental hygiene faculty. $\mathrm{Dhir}^{27}$ states in order to meet the challenge of increasing minority representation in the dental hygiene profession, dental hygiene program administrators and educators must increase efforts to recruit and admit qualified minority applicants. Dhir found non-hispanic whites comprised $89.5 \%$ of all dental hygiene students and $93.2 \%$ of all dental hygiene faculties. Minorities comprised the remaining $10.5 \%$ and $6.8 \%$ of dental students and faculty, respectively. Dhir concluded the small percentage of minority students and faculty in dental hygiene programs was due to a lack of knowledge about and interest in the dental hygiene profession, lack of academically qualified minority applicants, and lack of recruitment efforts by dental hygiene programs. $^{27}$

Curriculum changes within dental hygiene programs are a must in order to meet the demands of the diverse population. In Japan, dental hygiene education and associate programs 
are shifting from two to three year programs in order to meet the diverse needs of society. Health problems within a rapidly aging population require dental hygienist to provide client care that is not just highly professional but also individualized. ${ }^{29}$ To meet the need of the aging Japanese population, the Miyagi Advanced Dental Hygienist College developed a course in dysphagia management which was taught to third year students.

Recognizing the ever-increasing need for qualified, professionally competent, and an evolving profession of dental hygiene, the University of the Pacific in San Francisco, CA, USA has a three year accelerated Bachelor's degree program. The program is more patientcentered and follows a humanistic teaching approach with community-based experiential learning.

\section{Conclusions}

Students' clinical experiences with clients of diverse cultural backgrounds are dependent upon the cultural makeup of the community. Dhir found there was a moderately strong positive relationship between the ethnic/racial group composition of students in dental hygiene programs and the corresponding state population. ${ }^{27}$ However, there was a weak positive relationship between faculty in dental hygiene programs and the corresponding state population of the same ethnic/racial groups. ${ }^{27}$ In programs where the community's diversity is limited the program might arrange for exchange programs with other dental hygiene programs with a more culturally diverse clientele. Organizing community outreach focus groups, attending cultural events, and developing collegial relationships with other health professionals from various ethnic and minority groups would allow dental hygienists to become more familiar with different cultures and their oral health practices, attitudes, self care beliefs, and concerns. ${ }^{23}$

The current Dental School Accreditation Standard 2-17 states "graduates must be competent in managing a diverse patient population and have the interpersonal and communication skills to function successfully in a multicultural work environment". ${ }^{30}$ The Oral Health in America: A Report of the Surgeon General found there were disparities in oral health based on race, culture,

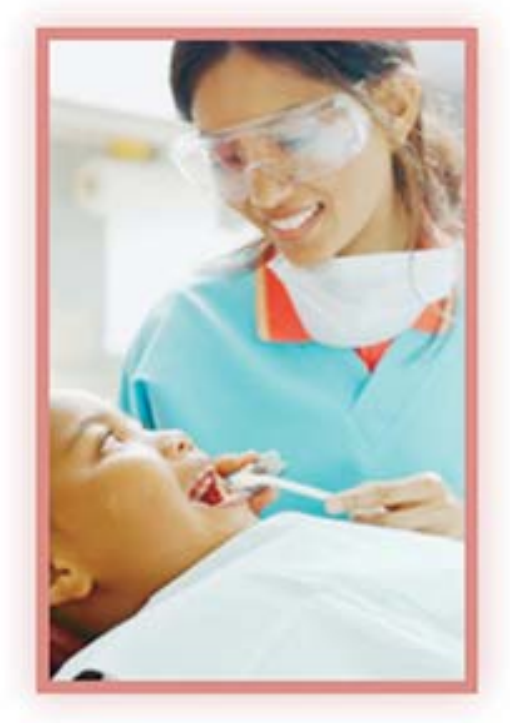

and ethnicity. ${ }^{31}$ For example, African-American males are diagnosed with oral cancer at a later point in the disease than whites, and the mortality rate for black men is far greater than it is for whites. The Institute of Medicine 's (IOM) report on Unequal Treatment found three factors unrelated to healthcare access to the differences in treatments and outcomes between minorities and non-minorities. First, there were inconsistent patient behaviors and attitudes related to compliance with treatment regimens, which attributed to cultural mismatch between minority patients and their providers. ${ }^{32}$

Secondly, according to the IOM study includes "provider bias (or prejudice) against minorities; greater clinical uncertainty when interacting with minority patients; and beliefs (or stereotypes) held by the provider about the behavior or health of minorities. ${ }^{\prime 31}$ And the last factor related to treatment differences between whites and minorities is mandated managed care, which disrupts patients who are accustomed to the continuity and personal relationship provided by traditional community-based providers. ${ }^{31}$ Given these realities, there must be a continued high level of concern for oral health services for minority communities. Public health community centers need to increase their local health departments to include a direct oral health education and service component. Long-term care facilities need to provide oral examinations and initiate prevention, education, and oral health treatment services to their residents more frequently. Dental and dental hygiene 
programs need to support their graduates in establishing or servicing practices in inner-cities or in underserved populations. Create licensure for an Advanced Dental Hygiene Practitioner (ADHP) that can provide oral health service to underserved populations without the current restrictions.

A substantial improvement in the diversity of the oral health student body and eventual workforce is a critical and necessary element in achieving the goals of improving oral health and quality of life and eliminating health disparities. Clients' values and general oral healthcare beliefs are rooted in culture. The current population demographics reveal an increasingly multicultural society; therefore, dental hygienists must be prepared to practice in a culturally diverse environment, in a sensitive and appropriate manner, in order to deliver optimal oral hygiene care.

\section{References}

1. Spector RE. Cultural Diversity in Health and Illness, 6th ed. Upper Saddle River, NJ: Prentice Hall; 2004; 1 -75.

2. The American Heritage Dictionary, 1976 Edition. New College: Boston, MA.

3. Nightingale, F. Notes on nursing - What it is, what it is not. Appleton and Co, 1860; New York

4. Murray, R., \& Zentner, J. Nursing concepts for health promotion. Englewood Cliffs, NJ: Prentice Hall, $1975 ; 1-4$.

5. Rogers, M. Nursing: A science of unitary human beings. Conceptual models for nursing practice 3rd ed. Norwalk, CT: Appleton \& Lange: 1989; 181-188.

6. Rosenstock, I. M. Why people use health services. Millbank Memorial Fund Quartely 1966; 44: 94-127.

7. Kozier, B., Erb, G., Blais, K., Wilkinson, J., and Van Leuven, K. Fundamentals of nursing concepts, process, and practice. Upper Saddle River, NJ: Prentice Hall 2000; 176.

8. Illness defined by Wikipedia Encyclopedia (www.http://en.wikipedia.org/wiki/lllness.com)

9. Darby ML, Walsh, MM. Dental Hygiene Theory and Practice 2nd ed. Philadelphia: Saunders 2003; 21-22, 59-73

10. Annual Estimates of the Population for the United States and States: April 1, 2000. www.census.gov

11. Code of Federal Regulations 3, Title 24 (Revised as of April 1, 2005)

12. Federal Register 65, no. 36 (2002, February 14), 6931-6933

13. Poverty in the United States: 2000 U.S. Census Bureau Current Population Reports Series. Washington DC: U.S. Government. www.census.gov/hhes/poverty/poverty00/table5.html

14. Centers for Disease Control and Prevention, National Center for Health Statistics, Healthy People 2000 Review, 1989-1999 (http://healthypeople.gov)

15. MacKay, A.P., Fingerhut, L.A., \& Duran, C.R. (2000). Adolescent health chartbook. Health, United States, 2000. Hyattsville, MD: National Center for Health Statistics.

16. Chen, M. A sociodemographic analysis of preventive dental behavior among white American families. Health Educ Quarterly, 1986 Summer; 13(2) : 105-15.

17. Ismail, A.I. \& Sohn, W. The impact of universal access to dental care on disparities in caries experience in children. J Amer Dent Assoc 2001 Mar; 132(3): 295-303.

18. Zimmerman, M., Bornstein, R., and Martinsson., T. Attitudes and knowledge about preventive dental care in Chilean refugees in Sweden. Community Dent Health 1993 Dec; 10(4): 343-51.

19. Cairns D. The role of the dental hygienist in a multicultural society: Review of literature. Probe 1993 Sept-Oct; 27(5): 177-81.

20. Tamparo CT \& Lindh WQ. Therapeutic Communication for Health Professionals, 2nd ed. Clifton Park, NJ: Delmare Leaning 2000; 29 -32.

21. Management Services \& U.S. Department of health and Human Services. The provider's guide to quality and culture. http://www.omhrc.gov

22. Beltran-Aguilar, E., Barker, L., Canto, M., Dye, B., Gooch, B., Griffin, S., Hyman, J., Jaramillo, F., Kingman, A., Raymer, R., Selwitz, R., and Tianxia Wu. Surveillance for dental caries, dental sealants, tooth retention, edentulous and enamel fluorosis - U.S. Department Center of Disease Control. 1999-2002. 
23. Sundby, A. \& Peterson, P.E. Oral health status in relation to ethnicity of children in the Municipality of Copenhagen, Denmark. Int J Paediatr Dent 2003 May; 13:150-7.

24. Fitch, P. Cultural Competence and Dental Hygiene Care Delivery: Integrating Cultural Care into the Dental Hygiene Process of Care. J Dent Hyg 2004 Winter ; 78(1): 11-21.

25. Commission on Dental Accreditation: Accreditation Standards for Dental Hygiene Education Programs. Chicago, American Dental Association, 1998; 22.

26. United States Department of Health and Human Services: Healthy People 2010. Washington, DC, U.S. Government. (www.healthypeople.gov)

27. Morey, DP \& Leung, JJ. The multicultural knowledge of registered dental hygienist: A pilot study. J Dent Hyg 1993 May-June; 67(4): 180-85

28. Dhir I, Tishk MN, Tira DE, Holt LA. Ethnic and racial minority students in U.S. entry-level dental hygiene programs: A national survey. J Dent Hyg 2002 Summer; 76(3): 193-201

29. Sato $Y$, Miura A, Saito A. Dysphagia management in a 3-year dental hygiene education programme in Japan. Int J Dent Hyg , 2005 Nov; 3(4): 179-84.

30. Commission on Dental Accreditation. Accreditation standards for dental education programs. Standard 2-17. Chicago: American Dental Association, July 1, 2002.

31. Noonan AS \& Evans CA. The Need for Diversity in the Health Professions. J Dent Educ 2003 Sept; 67: 1030-33.

32. Formicola AJ, Stavisky J, Lewy R. Cultural Competency: Dentistry and Medicine Learning from One Another. J Dent Educ 2003 Aug; 67(8): 869-75.

\section{About the Authors}
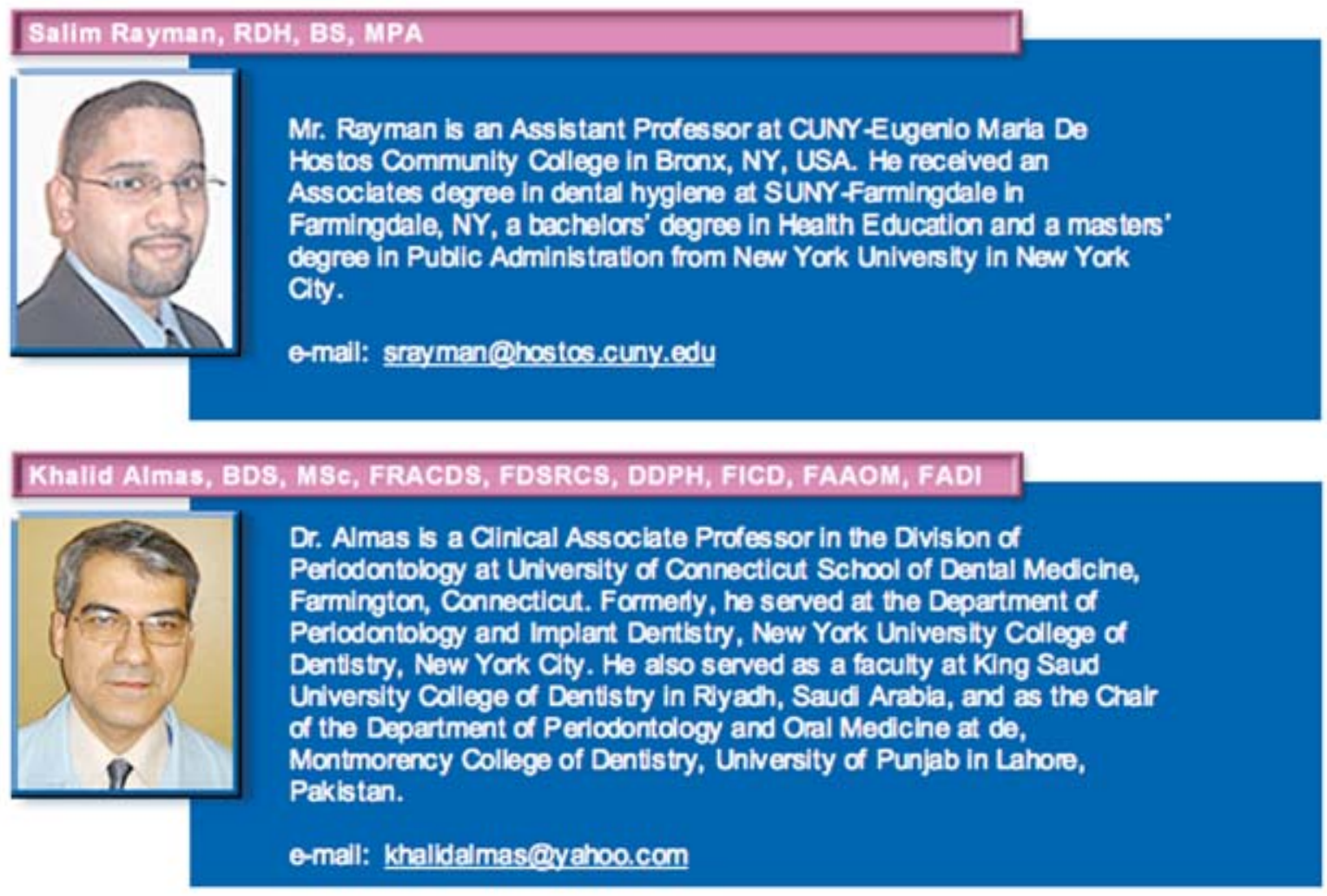